
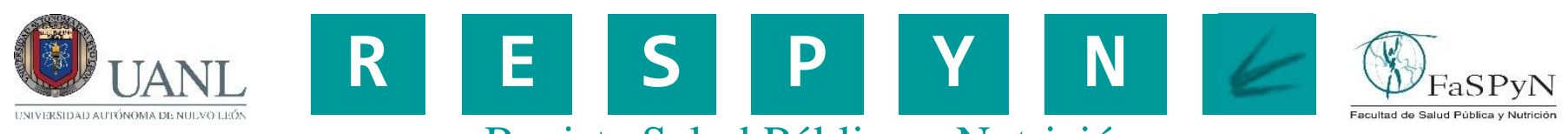

Revista Salud Pública y Nutrición

\title{
PERCEPCIÓN DEL CUERPO SALUDABLE Y SU RELACIÓN CON LA INSATISFACCIÓN CORPORAL EN ADOLESCENTES MEXICANOS
}

PERCEPTION OF THE HEALTHY BODY AND ITS RELATION TO BODY DISSATISFACTION IN MEXICAN ADOLESCENTS

Cruz Licea, Verónica ${ }^{1}$ Urbina Cedillo, Clara ${ }^{1}$ Alvear Galindo, María Guadalupe ${ }^{1}$ Ortiz Hernández, Luís ${ }^{2}$ Morán Álvarez, Isabel Cristina ${ }^{1}$

1 Departamento de Salud Pública de la Facultad de Medicina de la Universidad Nacional Autónoma de México. 2 Atención a la Salud, Universidad Autónoma Metropolitana Unidad Xochimilco, México.

Citation: Cruz Licea, V. Urbina Cedillo, C. Alvear Galindo, M.G. Ortiz Hernández, L. Morán Álvarez, I. C. (2018) Percepción del cuerpo saludable y su relación con la insatisfacción corporal en adolescentes mexicanos. Revista de Salud Pública y Nutrición, 17(1), 23-29.

Editor: Esteban G. Ramos Peña, Dr. CS., Universidad Autónoma de Nuevo León, Facultad de Salud Pública, Monterrey Nuevo León, México.

Copyright: (C2018 Cruz Licea, V. et al. This is an open-access article distributed under the terms of Creative Commons Attribution License [CC BY 4.0], which permits unrestricted use, distribution, and reproduction in any medium, provided the original author and source are credited.

Competing interests: The authors have declared that no competing interests exist.

DOI: https://doi.org/10.29105/respyn17.1-4

Recibido: 27 de febrero 2018; $\quad$ Aceptado: 10 de abril 2018

Email: veronica_cruzlicea@yahoo.com.mx 


\title{
PERCEPCIÓN DEL CUERPO SALUDABLE Y SU RELACIÓN CON LA INSATISFACCIÓN CORPORAL EN ADOLESCENTES MEXICANOS
}

\author{
Cruz Licea, Verónica ${ }^{1}$ Urbina Cedillo, Clara ${ }^{1}$ Alvear Galindo, María Guadalupe ${ }^{1}$ Ortiz Hernández, Luís ${ }^{2}$ Morán \\ Álvarez, Isabel Cristina ${ }^{1}$
}

1 Departamento de Salud Pública de la Facultad de Medicina de la Universidad Nacional Autónoma de México. 2 Atención a la Salud, Universidad Autónoma Metropolitana Unidad Xochimilco.

\section{RESUMEN}

Introducción: Las campañas de prevención de la obesidad que simbolizan el cuerpo sano sin exceso de peso podrían afectar la percepción de la imagen corporal de los adolescentes, como ocurre con el ideal estético. Objetivo: Evaluar su percepción del cuerpo sano y su relación con la insatisfacción corporal Métodos: Se realizó una encuesta en 460 adolescentes, estudiantes de una escuela pública en la Ciudad de México. Se utilizó una prueba de silueta para evaluar la percepción de: su imagen actual, la imagen que les gustaría tener y la imagen que consideraban saludable de acuerdo con las campañas de prevención de la obesidad. La información se analizó mediante correlaciones estadísticas entre cada una de las tres percepciones corporales y la insatisfacción. Resultados: La mayoría de los adolescentes (76.8\%) ubican al cuerpo sano en un índice de masa corporal de peso normal. Se encontró una relación casi nula con la dirección negativa entre el cuerpo sano y la insatisfacción corporal. Conclusión: Los resultados indican que las campañas que promueven el cuerpo sano logran que los adolescentes identifiquen un cuerpo asociado con menores riesgos para la salud, sin implicar una afectación importante en su percepción corporal.

Palabras Clave: prevención, obesidad, cuerpo sano, insatisfacción corporal.

\section{ABSTRACT}

Introduction: The obesity prevention campaigns that symbolize the healthy body without excess weight could affect the perception of the body image of adolescents, as it happens with the aesthetic ideal. Objective: Evaluate their perception of the healthy body and its relationship with body dissatisfaction. Methods: A survey was conducted in 460 adolescents, students of a public school in Mexico City. A silhouette test was used to assess the perception of: their current image, the image they would like to have, and the image they considered healthy according to the obesity prevention campaigns. The information was analyzed through statistical correlations between each of the three body perceptions and dissatisfaction. Results: The majority of adolescents (76.8\%) place the healthy body in a body mass index of normal weight. An almost null relationship with negative direction was found between the healthy body and body dissatisfaction. Conclusion: The results indicate that the campaigns that promote the healthy body achieve that adolescents identify a body associated with lower risks to health, without implying an important affectation in their corporal perception.

Key words: prevention, obesity, healthy body, body dissatisfaction 


\section{Introducción}

Desde hace dos décadas hasta el día de hoy, varias instituciones gubernamentales mexicanas han llevado acabo intensas campañas de prevención de la obesidad en medios de comunicación como una estrategia para disminuir su prevalencia en la población. A través de la transmisión de mensajes, pretenden motivar a las personas para que adopten un estilo de vida saludable, como disminuir el consumo de alimentos con alto contenido de grasas, sal y azúcares, aunado a realizar actividad física (Barquera, Rivera-Dommarco \& Gasca-García, 2001; Barquera, Campos-Nonato, Rojas \& Rivera, 2010). También hay mensajes que promueven mantenerse en un índice de masa corporal (IMC) de 18.5 a $25 \mathrm{~kg} / \mathrm{m}^{2}$ y una circunferencia de cintura menor a $80 \mathrm{~cm}$ en mujeres y $94 \mathrm{~cm}$ en hombres; estas medidas van a simbolizar al "cuerpo saludable".

En ocasiones utilizan imágenes de personas o caricaturas que describen a la obesidad con algunas de las siguientes características: cuerpo grande, redondo, abdomen voluptuoso, glotón, perezoso, gracioso, poco atractivo y enfermo. Mientras que el cuerpo saludable es simbolizado como: delgado, alto, luce feliz, activo, atractivo, sin grasa abdominal y con musculatura en el caso de imágenes masculinas (Asistencia, Asesoría y Administración de Espectáculos, 2013; Instituto Mexicano del Seguro Social, 2015; Secretaria de Salud de México, 2017; Secretaria de Salud de México, 2013; Secretaria de Salud del Distrito Federal, 2011). Con ello pretenden alentar a la población a que adopten ciertos tipos corporales y eviten otros.

Estas imágenes están reforzando los estereotipos negativos ya existentes de la obesidad, que provocan discriminación hacia las personas con obesidad. Las personas que son discriminadas se tornan más vulnerables a daños psicológicos y aumentan la probabilidad de llevar a cabo actividades que contribuyan a mantener o empeorar su situación, por ejemplo comer más o hacer dietas estrictas (Bermúdez \& Hernández, 2012; Ashmore, Friedman, Reichmann, \& Musante, 2008). Siendo los adolescentes los más afectados, debido a que están en una etapa de la vida de cambios y aceptación de la imagen corporal. Los adolescentes tienen mayor preocupación por su apariencia física y no desean ser rechazados por tener una apariencia diferente a lo valorado socialmente (Rodríguez \& Caño, 2012).
En la actualidad, el ideal corporal es caracterizado por un cuerpo esbelto y es significado de triunfo, éxito y belleza. Este ideal es impuesto por publicidad de la industria de la moda y del mercado de productos "milagro" que supuestamente disminuyen el exceso de peso sin esfuerzo (Toro, 1996). La influencia dañina que tiene este ideal estético en los adolescentes sobre la imagen corporal, y en especial en el desarrollo de trastornos de la conducta alimentaria ha sido ampliamente corroborada (Martínez-González, et al., 2003; Austin \& Smith, 2008). No obstante, el efecto que tiene la promoción de un cuerpo saludable como ausente de exceso de peso, es un tema controversial (Cohen, Perales \& Steadman, 2005; Ramos, 2015; O`Dea, 2005) y poco estudiado en nuestro país.

Hay evidencia de efectos negativos de campañas de prevención que han utilizado imágenes de cuerpos saludables o esbeltos en grupos específicos (Shentow-Bewsh, Keating \& Mills, 2016; Boutelle, Neumark-Sztainer, Story \& Resnick, 2002; Owena \& Spencer, 2013). Ejemplo de esto, es la intervención que se llevó acabo en mujeres adolescentes a quienes se les presentaron carteles con imágenes de personas delgadas como sanas. Se encontró que las adolescentes consideraron a las imágenes como un recordatorio de que no son perfectas y están imposibilitadas de alcanzar ese cuerpo, haciéndolas sentir inseguras de su peso y deseo de querer perder peso (O'Dea, 2002).

Otra intervención utilizó carteles promocionales con diferentes imágenes corporales y preguntó a mujeres con bulimia su opinión con respecto al peso saludable. Se encontró que la esbeltez la consideran como saludable y que la regulación cuidadosa de la ingesta y el ejercicio son prácticas saludables; así como las prácticas de comer compulsivamente y compensar (Burns \& Gavey, 2004). En hombres, el efecto negativo es semejante; una publicación que revisó varios estudios de hombres jóvenes encontró que la insatisfacción corporal aumenta cuando ven imágenes de hombres musculosos y atractivos como cuerpos ideales y saludables (Blond, 2008).

De acuerdo con lo anterior, se planteó como objetivo evaluar la percepción del cuerpo saludable en un grupo de adolescentes mexicanos y conocer su relación con la insatisfacción corporal. Con el fin de 
aportar información sobre el tamaño que tiene el cuerpo que los adolescentes consideran como saludable y su efecto negativo.

\section{Material y Métodos}

El estudio corresponde a un diseño descriptivo, correlacional y comparativo. Se realizó una encuesta durante el mes de septiembre y octubre de 2014. La población de estudio fueron adolescentes inscritos en la única escuela de nivel secundaria perteneciente a la Universidad Nacional Autónoma de México (UNAM). Se les invitó a participar a todos los estudiantes. Se incluyeron aquellos estudiantes que dieron su consentimiento verbal y entregaron el consentimiento firmado por los padres o tutores. En total, se estudiaron 460 adolescentes, $46 \%$ eran del sexo masculino y $54 \%$ femenino. El promedio de edad en años cumplidos fue $12.7 \pm 0.9$ años.

\section{Instrumentos y procedimiento}

A los adolescentes se les preguntaron datos generales que fueron tratados de forma individual $y$ confidencial. Se acondicionó una aula para realizar las mediciones antropométricas, se les midió el peso y la estatura utilizando una báscula digital (marca OMRON, modelo HBF-514) y un estadímetro de pared (marca SECA modelo 214) debidamente calibrados. Se calculó el IMC con el puntaje z para la edad y sexo de acuerdo con la clasificación de la Organización Mundial de la Salud (OMS): peso bajo $(\leq-2.00)$, peso normal (-1.99 a 0.99$)$, sobrepeso (1.00 a 1.99) y obesidad ( $\geq 2.00)$ (OMS, 2004).

Se utilizó el test de siluetas de Stunkard (Stunkard \& Album, 1981) para evaluar la percepción de la imagen corporal. A los adolescentes se les solicitó seleccionaran una de entre nueve siluetas o imágenes corporales que van de la más delgada hasta la más gruesa para hombres y mujeres considerando: 1) la imagen que refleja mejor su imagen corporal actual (nombrada para su análisis como percibida), 2) la imagen que les gustaría tener (nombrada como deseada) y 3) la imagen que consideran es el cuerpo saludable (nombrada como saludable) de acuerdo a las campañas de prevención de la obesidad.
Figura 1. Imágenes corporales de mujeres y hombres de acuerdo al índice de masa corporal.

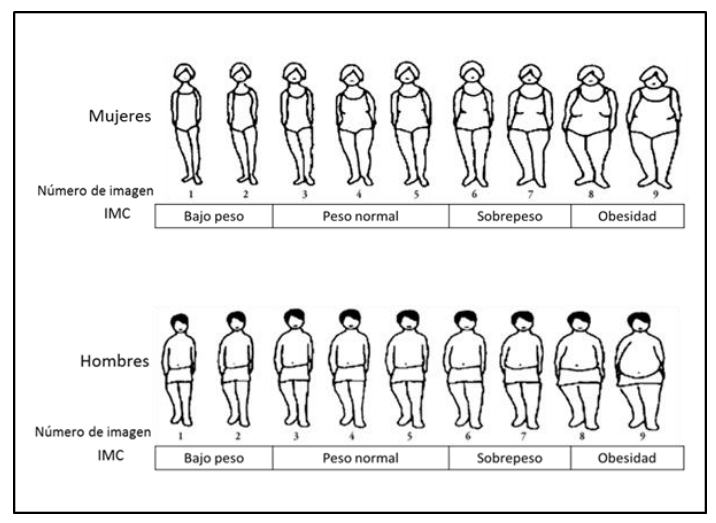

La insatisfacción corporal se consideró como la diferencia entre la imagen que refleja mejor su imagen corporal actual (percibida) y la imagen que les gustaría tener (deseada). Cuando el resultado fue un valor positivo se consideró como insatisfacción positiva que significa que desea estar más delgado, $\mathrm{y}$ cuando la diferencia resultó un valor negativo, se consideró como insatisfacción negativa que significa que desea tener más peso.

\section{Análisis estadístico}

La información se capturó y analizó en el programa SPSS para Windows versión 20.0. Se describieron con porcentajes las imágenes corporales seleccionadas como percibida, deseada y saludable. Se comparó por sexo, edad e IMC con la prueba chi cuadrada $\left(\mathrm{X}^{2}\right)$ a un límite de significación estadística $\mathrm{p}<0.05$.

Cada una de las imágenes seleccionadas (percibida, deseada y saludable), fueron clasificadas de acuerdo al IMC en: imagen 1 y 2 como bajo peso, 3 a 5 como peso normal, 6 y 7 como sobrepeso y por último, 8 y 9 como obesidad (Figura 1). Cabe mencionar que esta clasificación ha sido estandarizada y empleada exitosamente como indicador de autoreporte de IMC (Osuna-Rámirez, Hernández-Prado, Campuzano \& Salmerón, 2006). Renombrándolas para su análisis como: IMC percibido, IMC deseado y IMC saludable.

Posteriormente, se hizo un análisis de correlación mediante el coeficiente de Spearman ( $\left.\mathrm{r}_{\text {Spearman }}\right)$ de cada una de estas variables con la insatisfacción 
corporal, estratificando por sexo. El límite de significación estadística fue $\mathrm{p}<0.05$.

\section{Resultados}

De los 460 adolescentes, $2.8 \%(\mathrm{n}=13)$ tenían peso bajo, $55.0 \%(\mathrm{n}=253)$ peso normal, $17.0 \%(\mathrm{n}=78)$ sobrepeso y $25.2 \%(n=116)$ tenían obesidad. No se encontraron diferencias significativas estadísticamente entre el IMC evaluado por sexo y edad.

\section{Descripción de las imágenes percibida, deseada y saludable}

Las imágenes más seleccionadas como imagen percibida en las mujeres fueron $3(21.4 \%)$ y 4 (19.4\%), ambas corresponden a un IMC de peso normal; las imágenes menos seleccionadas fueron 9 $(0.0 \%)$ y $8(2.4 \%)$, ambas pertenecen a un IMC de obesidad. En los hombres, las imágenes más seleccionadas como imagen percibida fueron 3 $(22.6 \%)$ y $4(19.3 \%)$, ambas pertenecen a IMC de peso normal; mientras que las imágenes menos seleccionadas fueron $9(0.0 \%)$ y $8(1.4 \%)$, que pertenecen a IMC de obesidad.

De acuerdo con la imagen deseada, las mujeres seleccionaron mayormente las imágenes $3(31.9 \%)$ y $5(25.4 \%)$, ambas corresponden a un IMC de peso normal; y las menos seleccionadas fueron $9(0.0 \%)$, $8(0.4 \%)$ y $7(0.4 \%)$ que corresponden a un IMC de obesidad y sobrepeso. En los hombres, las imágenes más seleccionadas como deseadas fueron $3(36.8 \%)$ y $4(29.2 \%)$, ambas corresponden a un IMC de peso normal; y las menos seleccionadas fueron $9(0.0 \%)$, $8(0.5 \%)$ y $7(0.5 \%)$ que corresponden a un IMC de obesidad y sobrepeso.

Las imágenes más seleccionadas como cuerpo saludable por las mujeres fueron $3(28.6 \%)$ y 5 (27.4\%), ambas pertenecen a un IMC de peso normal, y las imágenes menos seleccionadas fueron $8(0.8 \%)$ y $7(1.2 \%)$ que corresponden a un IMC de obesidad y sobrepeso. Hubo mujeres que seleccionaron como saludable a las imágenes 1 $(4.4 \%)$ y $2(6.0 \%)$ que corresponden a un IMC de bajo peso.

Los hombres seleccionaron como cuerpo saludable las imágenes 4 (31.1\%) y 3 (27.8\%) que corresponden a un IMC de peso normal y las menos seleccionadas fueron $7(0.9 \%)$ y $9(1.9 \%)$ que corresponden a un IMC de obesidad y sobrepeso, respectivamente. Hubo hombres que seleccionaron como saludable a las imágenes $1(3.8 \%)$ y $2(9.9 \%)$ que corresponden a un IMC de bajo peso.

Análisis de correlación entre IMC evaluado, percibido, deseado y saludable

Al llevar acabo el análisis de correlación (Tabla 1), se encontró una relación positiva significativa estadísticamente entre IMC evaluado e IMC percibido $\left(\mathrm{r}_{\text {Spearman }}=0.598 ; \mathrm{p}=0.000\right), \mathrm{IMC}$ evaluado e IMC deseado $\left(\mathrm{r}_{\text {Spearman }}=0.130 ; \mathrm{p}=0.005\right)$, $\mathrm{y}$ entre IMC percibido e IMC deseado ( $\mathrm{r}_{\text {Spearman }}=0.437$; $\left.\mathrm{p}=0.000\right)$. En el caso del IMC saludable, se encontró correlación con el IMC percibido $\left(\mathrm{r}_{\text {Spearman }}=0.161\right.$; $\mathrm{p}=0.001)$ y deseado $\left(\mathrm{r}_{\text {Spearman }}=0.334 ; \mathrm{p}=0.000\right)$. No se encontró correlación significativa estadísticamente entre el IMC evaluado con el IMC saludable.

En cuanto a las correlaciones estratificadas por sexo, en las mujeres se encontraron coeficientes ligeramente mayores a los calculados en los hombres, a excepción de la correlación entre el IMC evaluado y percibido $\left(\mathrm{r}_{\text {Spearman }}=0.645 ; \mathrm{p}=0.000\right)$.

\begin{tabular}{|c|c|c|c|c|c|c|}
\hline & \multicolumn{2}{|c|}{ IMC Percibido } & \multicolumn{2}{|c|}{ IMC Deseado } & \multicolumn{2}{|c|}{ IMC Saludable } \\
\hline & rSpearman & $\mathrm{p}$ & rSpearman & $\mathrm{p}$ & rSpearman & $\mathrm{p}$ \\
\hline IMC Evaluado & 0.598 & 0.000 & 0.130 & 0.005 & 0.012 & 0.795 \\
\hline Mujer & 0.574 & 0.000 & 0.139 & 0.028 & 0.012 & 0.85 \\
\hline Hombre & 0.645 & 0.000 & 0.128 & 0.063 & 0.024 & 0.725 \\
\hline IMC Percibido & -- & -- & 0.437 & 0.000 & 0.161 & 0.00 \\
\hline Mujer & -- & -- & 0.471 & 0.000 & 0.188 & 0.003 \\
\hline Hombre & -- & -- & 0.392 & 0.000 & 0.120 & 0.082 \\
\hline IMC Deseado & -- & -- & -- & -- & 0.334 & 0.000 \\
\hline Mujer & -- & -- & -- & -- & 0.407 & 0.000 \\
\hline Hombre & -- & -- & -- & -- & 0.240 & 0.000 \\
\hline
\end{tabular}

\section{Insatisfacción corporal y el cuerpo saludable}

La frecuencia de insatisfacción corporal entre los adolescentes fue $47.0 \%$, de los cuales el $72.7 \%$ está insatisfecho porque desearían estar más delgado (insatisfacción positiva) y $27.3 \%$ desearía tener más peso (insatisfacción negativa). Por sexo, se encontró mayor frecuencia de satisfacción corporal entre las mujeres $(37.5 \%)$ que en los hombres $(33.0 \%)$, sin embargo esta diferencia no fue significativa estadísticamente. 
Al correlacionar IMC saludable con la insatisfacción positiva se encontró una dirección negativa casi nula $\left(\mathrm{r}_{\text {Spearman }}=-0.167 ; \mathrm{p}<0.05\right)$; esto es, disminuye su deseo de estar delgado cuando seleccionan como cuerpo saludable una imagen con mayor peso corporal. Mientras que la correlación del cuerpo saludable con la insatisfacción negativa, se encontró también una dirección negativa débil $\left(\mathrm{r}_{\text {Spearman }}=-\right.$ 0.279; $\mathrm{p}<0.05$ ), lo cual indica que su deseo por tener más peso corporal disminuye cuando el cuerpo saludable tiene menor peso. Al estratificar estas mismas relaciones por sexo, no se encontraron diferencias significativas estadísticamente.

\section{Discusión}

Entre los resultados de esta investigación hubo datos esperados que coincide con la literatura, como la fuerte relación entre el IMC evaluado y percibido, lo que reafirma que se puede utilizar siluetas corporales como indicador de autoreporte de IMC cuando no es factible medir directamente el peso y la estatura. También en concordancia con la literatura, se encontró una diferencia entre el IMC evaluado y deseado, se conoce que la mayoría de las personas desean tener menor peso del que tienen (Morán, Cruz-Licea \& Iñarritú, 2007). Finalmente, los resultados coinciden con investigaciones publicadas en los últimos años, que refieren que cada día hay más hombres que presentan problemas relacionados con la imagen corporal y el control del peso, de aquí que fueron escasas las diferencias halladas por sexo (Vázquez, López, Álvarez, Mancilla \& Oliva, 2006; Saucedo-Molina, Escamilla-Talón, Portillo-Noriega, Peña-Irecta \& Calderón-Ramos, 2008).

En cuanto a los nuevos hallazgos de esta investigación, se detectó que ocho de cada diez adolescentes refirieron como cuerpo saludable aquella imagen correspondiente a un IMC de peso normal. Lo que indica que los adolescentes reconocen el cuerpo que es señalado por las campañas de prevención de la obesidad como aquel de menor riesgo para la salud. Sin embargo, se observó que alrededor de una décima parte de los adolescentes seleccionaron como cuerpo saludable imágenes correspondientes a un IMC de bajo peso, lo cual es preocupante porque creen que la delgadez es saludable. Esto plantea la interrogante sobre si los adolescentes seleccionaron esa imagen corporal porque la consideraron de menor riesgo a la salud, o bien, porque esa imagen es el ideal de acuerdo a los estándares dominantes de belleza.

También se encontró que el cuerpo seleccionado como saludable no se relaciona con las medidas de su cuerpo. Esto es, las campañas de prevención de la obesidad logran que los adolescentes identifiquen un cuerpo asociado con menores riesgos a la salud, pero no implica que logren un cambio para que los adolescentes adopten ese peso corporal. La prevención deja de lado las fuertes contradicciones por las que pasan los adolescentes, por un lado viven en un ambiente obesogénico, y por otro lado, está el ideal de belleza donde predomina la delgadez; ambas situaciones van a determinar el comportamiento (Meléndez, Cañez \& Frías, 2012).

Así mismo, los adolescentes refirieron no percibirse como saludables y sólo algunos desearían serlo. Lo que plantea la necesidad de diseñar o rediseñar estrategias dirigidas exclusivamente a los adolescentes que tengan mayor impacto, ya que la "guerra contra la obesidad" está enfocada a la infancia y los adultos. Aunado a las acciones de prevención, el gobierno debe garantizar la disponibilidad, accesibilidad y calidad de alimentos y agua para consumo, además deben asegurar las condiciones para la práctica de actividad física para combatir a la obesidad (Panier, 2009; Gracia, 2010).

Por último, no se encontró que la imagen del cuerpo saludable afecte la percepción que tienen de su imagen corporal, ya que no se encontró relación con la insatisfacción corporal. Al parecer aunque los adolescentes saben que un cuerpo saludable es aquel sin exceso de peso corporal, no los afecta no tener ese cuerpo.

El estudio tuvo limitantes debido al tamaño y la selección de muestra estudiada. Se propone para futuras investigaciones abordar las necesidades, motivaciones y estímulos entre los adolescentes sobre el cuerpo saludable. Los hallazgos aquí presentados aportan información para la discusión sobre el enfoque del cuerpo saludable como estrategia de promoción de la salud y prevención de la obesidad, que resulta inquietante y éticamente cuestionable (O'Hara \& Gregg, 2006). Debido a que el cuerpo es utilizado como una estrategia política de poder (Foucault, 1990), al establecer estrictas normas sociales: ¿qué comer?, ¿cuánto ejercicio hacer?, 
¿cuánto pesar?, ¿qué cuerpo tener?; lo cual transgrede la diversidad y autonomía individual.

\section{Conclusiones}

Las campañas de prevención gubernamentales han logrado que los adolescentes identifiquen aquel cuerpo asociado con menores riesgos a la salud (sin exceso de peso corporal), sin implicar gran afectación en la satisfacción que tienen de la imagen corporal. Por lo que es importante considerar que cuando se diseñe una campaña de prevención de la obesidad, se eviten imágenes que fomenten estereotipos negativos de la obesidad que puedan dañar la autoestima y la satisfacción que se tenga del cuerpo.

\section{Agradecimientos:}

Se agradece a las autoridades escolares y a la Lic. Josefina del Carmen Plascencia González por el apoyo otorgado. Así como al Ing. David Limón Cruz por la elaboración del cuestionario electrónico. El documento forma parte de la tesis de la autora principal para obtener el grado de Doctor en Salud Colectiva de la Universidad Autónoma Metropolitana.

\section{Bibliografía}

Ashmore, J.A., Friedman, K.E., Reichmann, S.K., \& Musante, G.J. (2008). Weight-based stigmatization, psychological distress, \& binge eating behavior among obese treatment-seeking adults. Eating Behaviors, 9(2), 203-209.

Asistencia, Asesoría y Administración de Espectáculos. (2013). Lucha Libre contra la obesidad. 1, 2, 3 saludable otra vez. Recuperado de: http://www.gob.mx/salud/prensa/inicia-la-campanacontra-obesidad-1-2-3-saludable-otra-vez10484? idiom $=$ es

Austin, J.L., \& Smith, J.E. (2008). Thin ideal internalization in Mexican girls: A test of the sociocultural model of eating disorders. International Journal of Eating Disorders, 41, 448-457.

Barquera, S., Campos-Nonato, I., Rojas, R., \& Rivera, J. (2010). Obesidad en México: epidemiologia y políticas de salud para su control y prevención. Gaceta Médica de México, 146, 397-407.
Barquera, S., Rivera-Dommarco, J., \& Gasca-García, A. (2001). Políticas y programas de alimentación y nutrición en México. Salud Pública de México, 43(5), 464-477.

Bermúdez, B., \& Hernández, F. (2012). La estigmatización del cuerpo obeso: percepción en un grupo de adolescentes mexicanos. Archivos en Medicina Familiar, 14(1), 21-27.

Blond, A. (2008). Impacts of exposure to images of ideal bodies on male body dissatisfaction: A review. Body Image, 5, 244-250.

Boutelle, K., Neumark-Sztainer, D., Story, M., \& Resnick, M. (2002). Weight control behaviors among obese, overweight, and nonoverweight adolescents. Journal of Pediatric Psychology, 27(6), 531-540.

Burns, M., \& Gavey, N. (2004). "Healthy weight" at what cost? "bulimia" and a discourse of weight control. Journal of Health Psychology, 9, 549-565.

Cohen, L., Perales, D., \& Steadman, C. (2005). The O Word: Why the Focus on Obesity is Harmful to Community Health. Californian Journal of Health Promotion, 3, 154-161.

Foucault, M. (1990). La vida de los hombres infames. Ensayos sobre desviación y dominación. España: La Piqueta.

Gracia, M. (2010). La obesidad como enfermedad, la obesidad como problema social. Gaceta Médica de México, 146, 389-396.

Instituto Mexicano del Seguro Social. (2015). Chécate, mídete, muévete. Recuperado de: http://checatemidetemuevete.gob.mx/

Martínez-González, M.A., Gual, P., Lahortiga, F., Alonso, Y., Irala-Estévez, J., \& Cervera, S. (2003). Parental factors, mass media influences, and the onset of eating disorders in a prospective population-based cohort. Pediatrics, 111, 315-320.

Meléndez, J.M., Cañez, G.M., \& Frías, H. (2012). Comportamiento alimentario durante la adolescencia. ¿Nueva relación con la alimentación y el cuerpo? Revista Mexicana de Investigación en Psicología, 4 , 99-111.

Morán, C., Cruz-Licea, V., \& Iñarritú, M.C. (2007). El índice de masa corporal y la imagen corporal percibida como indicadores del estado nutricional en 
universitarios. Revista de la Facultad de Medicina UNAM, 50(2), 76-79.

O'Hara, L., \& Gregg, J. (2006). The war on obesity: a social determinant of health. $\mathrm{He}$

alth Promotion Journal of Australia, 17(3), 260-262.

O'Dea, J. (2002). Can body image education programs be harmful to adolescent females?. Eating Disorders, 10, $1-13$.

O'Dea, J. (2005). Prevention of child obesity: 'First, do no harm'. Health Education Research, 20, 259-265.

Organización Mundial de la Salud. (2004). Estrategia global sobre dieta, actividad física y salud. Recuperado de: http://apps.who.int/gb/ebwha/pdf_files/WHA57/A57 R17-sp.pdf.

Osuna-Ramírez, I., Hernández-Prado, B., Campuzano, J.C., \& Salmerón, J. (2006). Body mass index and body image perception in a Mexican adult population: the accuracy of selfreporting. Salud Pública de México, 48, 94-103.

Owena, R., \& Spencer, R. (2013). Body ideals in women after viewing images of typical and healthy weight models. Body Image, 10, 489-494.

Panier, H. (2009). La salud como consumo. La construcción del cuerpo saludable como dispositivo biopolitico en la Sociedad de Control. Trabajo presentado en V Jornadas de Jóvenes Investigadores. Buenos Aires, Argentina. Recuperado de: http://www.aacademica.org/000-089/208.pdf.

Ramos, X. (2015). The ineffectiveness and unintended consequences of the public health war on obesity. Canadian Journal of Public Health, 106, e79-e81.

Rodríguez, C., \& Caño, A. (2012). Autoestima en la adolescencia: análisis y estrategias de intervención. International Journal of Psychology and Psychological Therapy, 12(3), 389-403.

Saucedo-Molina, T.J., Escamilla-Talón, T.A., PortilloNoriega, I.E., Peña-Irecta, A., \& Calderón-Ramos, Z. (2008). Distribución e interrelación de factores de riesgo asociados a trastornos de la conducta alimentaria en púberes hidalguenses, hombres y mujeres, de 11 a 15 años de edad. Revista de Investigación Clínica, 60(3), 231-240.

Secretaria de Salud de México. (2013). Cinco pasos para la salud. Recuperado de: http://www.5pasos.mx/

Secretaria de Salud de México. (2017). Resta kilos, suma vida.

Recuperado https://www.gob.mx/insalud/articulos/obesidad-ysobrepeso?idiom $=\mathrm{es}$

Secretaria de Salud del Distrito Federal. (2011). No a la obesidad. Recuperado de: http://www.noalaobesidad.df.gob.mx/

Shentow-Bewsh, R., Keating, L., \& Mills, J. (2016). Effects of anti-obesity messages on women's body image and eating behavior. Eating Behaviors, 20, 4856.

Stunkard, A., \& Album, J. The accuracy of self-reported weights. American Journal of Clinical Nutrition, 34(8), 1593-1599.

Toro, J. (1996). El cuerpo como delito. Anorexia, bulimia, cultura y sociedad. Barcelona, España: Editorial Ariel.

Vázquez, R., López, X., Álvarez, G., Mancilla, J.M., \& Oliva, A. (2006). Insatisfacción corporal e influencia de los modelos estéticos en niños y jóvenes varones mexicanos. Enseñanza e Investigación en Psicología, 11(1), 185-197. 